\title{
COVID-19 vaccine safety and nocebo-prone associated hesitancy in patients with systemic rheumatic diseases: a cross-sectional study
}

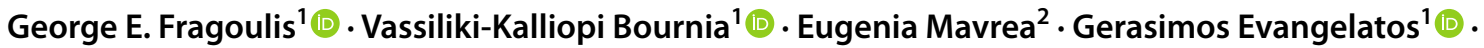 \\ Kalliopi Fragiadaki ${ }^{1} \cdot$ Anastasios Karamanakos $^{1} \cdot$ Eyrydiki Kravariti $^{1} \cdot$ Katerina Laskari $^{1}$ (I) $\cdot$ Stylianos Panopoulos ${ }^{1}$ (i)

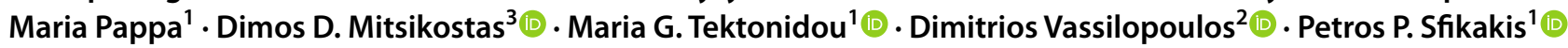

Received: 15 September 2021 / Accepted: 22 October 2021 / Published online: 5 November 2021

(c) The Author(s), under exclusive licence to Springer-Verlag GmbH Germany, part of Springer Nature 2021

\begin{abstract}
Objective To describe the rate and type of adverse effects (AEs) and the frequency of disease flares after COVID-19 vaccination and to assess the reasons for vaccination hesitancy (non-vaccination) in SRD patients.

Methods Telephone interviews were conducted of SRD patients consecutively enrolled (15/06/2021-1/7/2021). Participants were asked about the type of AEs and disease flare after vaccination. Reasons for vaccination hesitancy were recorded. Univariate and mutivariable analyses examined associations of demographic, clinical and other features, with occurrence of AEs, disease flare and non-vaccination. For the latter, association with negative vaccination behaviour (not influenza vaccinated for the last 2 years) and nocebo-prone behaviour (denoting AEs attributed to negative expectations [Q-No questionnaire]) was also tested.

Results 561 out of 580 contacted patients were included in the study. 441/561 (78.6\%) patients were vaccinated [90\% (Pfizer, Moderna), 10\% (Astra-Zeneca)]. AEs were reported by 148/441 (33.6\%), with rates being comparable between the three vaccines. AEs were more common in females and those with chronic obstructive pulmonary disease [OR, 95\% CI; females: 2.23 (1.30-3.83); COPD: 3.31 (1.24-8.83)]. Disease flare was reported in 9/441 (2\%) patients. For those unvaccinated, fear that the vaccine would be harmful (53.3\%), could cause disease flare (24.2\%) and/or could cause thrombosis (21.7\%) were the main reasons to do so. Multivariable analysis identified as independent variables for non-vaccination: nocebo-prone behaviour (OR; 95\% CI, 3.88; 1.76-8.55), negative vaccination behaviour $(6.56 ; 3.21-13.42)$ and previous COVID-19 infection $(2.83 ; 1.13-7.05)$. Higher educational status was protective $(0.49 ; 0.26-0.92)$.

Conclusion No new safety signals for COVID-19 vaccination were observed. Vaccination campaign should target SRD patients with nocebo-prone and negative influenza vaccination behaviour.
\end{abstract}

Keywords COVID-19 · Vaccination · Adverse effects · Disease flare

\section{Introduction}

COVID-19 has changed our clinical practice, producing also significant morbidity and mortality for all people, including those with systemic rheumatic disease (SRD) [1]. In this battle, vaccination is the main weapon in our arsenal, with many different types of vaccines produced [2]. Vaccination seems to be effective in the general population, as well as in SRD patients [3-7]. However, a considerable number of SRD patients remain unvaccinated, as vaccine hesitancy

George E. Fragoulis

geofragoul@yahoo.gr

Extended author information available on the last page of the article (reluctance for vaccinations) seems to be a problem amid the COVID-19 era and beyond [8,9]. The concerns raised for COVID-19 vaccines mainly pertain to the new technology used for the production of some of the approved vaccines, but also to the notion that vaccination can be related with disease flare and with adverse effects (AEs) [10-12].

The prompt identification of the reasons for vaccine hesitancy, as well as of factors that are associated with decreased vaccination uptake is of paramount importance. Also, the characteristics and the rate of AEs after vaccination are not well described in this subpopulation, with most $[4,5,13]$ but not all $[14,15]$ of the studies pertaining to mRNA-based vaccines. Along the same lines, disease flare after vaccination, which is sometimes a concern for patients with SRD 
[10-12], has been examined thoroughly, mainly in patients who received the Pfizer (BNT162b2) vaccine [3].

In our study we present data on safety and hesitancy about COVID-19 vaccination in a large, real-world cohort of patients with a variety of SRDs, who received three different types of vaccines. We comparatively describe the rate and type of AEs as well as the rate of disease flare, after vaccination against COVID-19 and explore any possible association between their occurrence and demographic, clinical, or other factors. For those who were not vaccinated, we report the reasons for vaccine hesitancy and examine whether there is any association with nocebo-prone and/or other behaviours.

\section{Patients and methods}

\section{Study population and recorded parameters}

Telephone interviews were conducted from 15/06/2021 to $1 / 7 / 2021$ by rheumatologists or rheumatology nurses from two tertiary hospitals ("Laiko" and "Hippokration" hospital), both referral centers for SRD. Patients with an SRD followed up in our outpatient rheumatology clinics were consecutively enrolled, following an alphabetical order of their surname as recorded in our files. The following features were recorded: COVID-19 infection (confirmed by polymerase chain reaction test), vaccination status against COVID-19, age, gender, disease duration, level of education (primary, secondary, university/college), employment status, co-morbidities on the basis of clinical diagnosis by treating physician or medication received (hypertension, coronary heart disease, congestive heart failure, diabetes mellitus, chronic kidney disease and chronic obstructive pulmonary disease [COPD]) and current treatment (corticosteroids, conventional disease modifying antirheumatic drugs [DMARDs], synthetic or biologic DMARDs) for SRD. For those vaccinated (having completed both doses of vaccination), the following features were recorded: type of vaccine received, experience of disease flare (based on their opinion) in the next 15 days after vaccination, whether they have discontinued any of their medication due to COVID-19 vaccination and type of AE (new symptom or sign) within 10 days after COVID-19 vaccination. The latter question was open, without answers being predefined. The severity of AE was not recorded.

In case they were not vaccinated, reasons for this decision were recorded. Predefined possible answers were as follows: belief that the vaccine would not be helpful, fear that vaccine could be harmful, fear of a disease flare after the vaccine, fear of thrombosis after the vaccine, vaccination against COVID-19 has not been suggested by their treating rheumatologist, previous infection with COVID-19, other reasons (e.g. allergies, treatment with rituximab). They were also asked if they missed any appointment with their treating rheumatology team over the last year and if they were willing to reconsider their decision about not getting the vaccine. Vaccination status for influenza for the last 2 years 2019 and 2020 has also been retrieved from our files. "Negative vaccination behaviour" was arbitrarily defined as not been vaccinated against influenza for the last 2 years (despite being recommended). Nocebo-prone behaviour has also been recorded in the patients enrolled in our study. This was defined based on the Q-No questionnaire (with a cutoff $>15$ considered as nocebo-prone behaviour), as previously described [16, 17]. Finally, data about treatment discontinuation during the first wave of COVID-19 pandemic (treatment non-adherence, partially previously published [18]) were retrieved.

It should be mentioned that, in our country, vaccination was offered for all SRD patients, irrespective of treatment, from March 2021 and onward. It was free of cost and only patients with previous anaphylactic shock were potentially excluded.

This study was approved by the Scientific Council of the "Laiko" hospital (no: 4.14/20, April 2020). Informed consent (verbal due to nature of the study) was obtained from all participants.

\section{Statistical analysis}

Continuous variables were expressed as mean $( \pm$ standard deviation, SD) and categorical variables as percentages (\%). Two-sided Fisher's exact and Mann-Whitney tests were used to compare categorical and continuous characteristics, respectively. Freeman-Halton extension of the Fisher exact probability test was used for comparison between the three different groups of vaccines.

Binary logistic regression analysis was performed using "Occurrence of adverse events" after vaccination against COVID-19 as dependent variable and sex, age, type of SRD, treatment received for SRD, unemployment, level of education, smoking status (current), disease duration, type of vaccine received, presence of arterial hypertension, coronary heart disease, congestive heart failure, diabetes mellitus, chronic kidney disease and COPD as independent variables. In another model aiming to predict "disease flare", sex, age, type of SRD, treatment received for SRD, type of vaccine administered and treatment discontinuation for COVID-19 vaccination served as independent variables. Finally, a third model was employed using "non-vaccination" as the dependent variable, and age, gender, educational status, employment status, comorbidities (arterial hypertension, coronary heart disease, congestive heart failure, diabetes mellitus, chronic kidney disease and COPD), medication for SRD, COVID-19 infection before start of the vaccination period, negative vaccination behaviour and nocebo-prone behaviour 
as independent variables. Statistical package SPSS 21.0 was used.

\section{Results}

\section{Cohort description}

In total, 580 patients were contacted. From them 14 were excluded from the study because they had not received both doses of vaccines and five did not participate in the study for various reasons. 561 patients were finally included in our study (75\% females, with a mean disease duration of approximately 10 years, Table 1$)$. Most, suffered from inflammatory arthritis $(n=326,58.1 \%)$ or connective tissue diseases $(n=154,27.5 \%)$, while $22(3.9 \%)$ and $59(10.5 \%)$ had a diagnosis of autoinflammatory disease or vasculitis, respectively. All, but 23, were on immunosuppressives as depicted in Table 1. About one-fifth were unemployed and about $30 \%$ had college/university education (Table 1 ). Three hundred and ninety-four out of $561(70.2 \%)$ reported that they had contacted our rheumatology team by telephone at least once during the COVID-19 pandemic.

\section{Vaccination uptake rate}

In total, $441 / 561(78.6 \%)$ of our patients were vaccinated against COVID-19. Mean \pm SD time from the time of the second dose to the time of the interview was $44.8 \pm 22.3$ days. Most of them received an mRNA-based

Table 1 Demographic characteristics of the patients included in the study

\begin{tabular}{ll}
\hline Characteristics & $n=561$ \\
\hline Female Gender, $n(\%)$ & $424(75.6 \%)$ \\
Age, mean \pm SD & $54.4 \pm 14.8$ \\
Smoking (current), $n(\%)$ & $151(26.9 \%)$ \\
Disease & \\
Inflammatory arthritis, $n(\%)$ & $326(58.1)$ \\
Connective tissue diseases, $n(\%)$ & $154(27.5)$ \\
Autoinflammatory, $n(\%)$ & $22(3.9)$ \\
Vasculitis, $n(\%)$ & $59(10.5)$ \\
Disease duration (years), mean \pm SD & $9.8 \pm 8.1$ \\
College/university level of education, $n(\%)$ & $207(36.8)$ \\
Unemployment, $n(\%)$ & $101(18.0)$ \\
Treatment & \\
On steroids, $n(\%)$ & $201(35.8)$ \\
On cDMARDs, $n(\%)$ & $362(64.5)$ \\
On b/tsDMARDs, $n(\%)$ & $313(55.8)$ \\
\hline
\end{tabular}

cDMARDS conventional disease-modifying antirheumatic drugs, $b /$ tsDMARDs biologic or targeted synthetic DMARDs vaccine [Pfizer, $n=380$ (86.2\%); Moderna, $n=14$ (3.2\%)], while $44(10.0 \%)$ had an adenovirus-based vaccine manufactured by Astra-Zeneca and $3(0.7 \%)$ from Johnson \& Johnson (Table 2).

\section{Adverse effects}

One hundred and forty-eight out of 441 (33.6\%) patients had adverse effects following the first [107/441 (24.3\%)], the second [108/441 (24.5\%)] or both doses [67/441 (15.2\%)] of vaccination. No differences were observed across different vaccines, the rate of AEs being as follows: Pfizer: 126/380 (33.2\%), Astra-Zeneca 14/45 (31.1\%), Moderna 6/14 (42.9\%), Johnson \& Johnson 2/2 (100\%). Of note, Astra-Zeneca vaccine had double the rate of AEs in the first compared to the second dose (24.4\% vs. $13.3 \%)$. In contrast, Pfizer demonstrated similar AE rates between the first and second dose (24.2\% vs. $26.0 \%)$ This was also the case for the 14 patients receiving the Moderna vaccine (28.6\% for both).

Fatigue, local pain at the site of injection and fever were the three most common AEs, followed by a variety of clinical features and laboratory abnormalities, as displayed in Table 3. One could note that local pain and fatigue were more commonly reported by patients receiving the Pfizer vaccine, compared to those receiving the Astra-Zeneca ( $17.1 \%$ vs. $2.3 \%$ and $19.5 \%$ vs. $11.4 \%$, respectively), while the opposite was the case for fever (10.8\% vs. $29.6 \%$ ).

AEs were more common in females (male gender: $23.6 \%$ vs. $36.7 \%$ female gender: $p=0.013$ ) and in those with COPD compared to those without (57.8\% vs. $32.5 \%, p=0.027$ ). Both variables remained statistically significant in the multivariable analysis [OR, 95\% CI; female gender: 2.23 (1.30-3.83); COPD: 3.31 (1.24-8.83)].

Table 2 Vaccination characteristics of patients included in the study

\begin{tabular}{ll}
\hline COVID-19 vaccinated & $n=441$ \\
\hline Vaccine & \\
Pfizer, $n(\%)$ & $380(86.2)$ \\
Moderna, $n(\%)$ & $14(3.2)$ \\
Astra-Zeneca, $n(\%)$ & $44(10.0)$ \\
Johnson \& Johnson, $n(\%)$ & $3(0.7)$ \\
Adverse effects, rates & $148 / 441$ \\
1st dose, $n(\%)$ & $107(24.3)$ \\
2nd dose, $n(\%)$ & $108(24.5)$ \\
Both doses, $n(\%)$ & $67(15.2)$ \\
Disease flare, rates & \\
1st dose, $n(\%)$ & $1(0.23)$ \\
2nd dose, $n(\%)$ & $8(1.81)$ \\
\hline
\end{tabular}

Type of vaccine received, rates of adverse effects and disease flare 
Table 3 Adverse effects reported after COVID-19 vaccination in 441 patients with systemic rheumatic disease

\begin{tabular}{|c|c|c|c|c|c|}
\hline & \multicolumn{4}{|c|}{$N, 1$ st dose $/ 2$ nd dose ( $\%$ first dose $/ \%$ second dose) } & $\begin{array}{l}\text { Total } \\
\text { doses } \\
(n=880)\end{array}$ \\
\hline Fatigue & $4 / 1(9.1 / 2.3)$ & $38 / 36(10.0 / 9.5)$ & $0 / 3(0 / 2.1)$ & 1/NA (33.3/NA) & $83(9.4)$ \\
\hline Local pain & $1 / 0(2.3 / 0)$ & $33 / 32(8.7 / 8.4)$ & $2 / 2(14.3 / 14.3)$ & 1/NA (33.3/NA) & $71(8.1)$ \\
\hline Fever & $8 / 5(18.2 / 11.4)$ & $12 / 29(3.2 / 7.6)$ & $1 / 2(7.1 / 14.3)$ & 1/NA (33.3/NA) & $58(6.6)$ \\
\hline Headache & $1 / 0(2.3 / 0)$ & $16 / 19(4.2 / 5.0)$ & $1 / 0(7.1 / 0)$ & 0/NA (0/NA) & $37(4.2)$ \\
\hline Dizziness & $1 / 1(2.3 / 2.3)$ & $13 / 7(3.4 / 1.8)$ & $0 / 0(0 / 0)$ & 0/NA (0/NA) & $22(2.5)$ \\
\hline Myalgias/arthralgias & $1 / 0(2.3 / 0)$ & $5 / 11(1.3 / 2.9)$ & $1 / 0(7.1 / 0)$ & 1/NA (33.3/NA) & $19(2.2)$ \\
\hline Rash & $0 / 0(0 / 0)$ & $6 / 2(1.6 / 0.5)$ & $0 / 0(0 / 0)$ & 0/NA (0/NA) & $8(0.91)$ \\
\hline Chills & $1 / 1(2.3 / 2.3)$ & $0 / 3(0 / 0.8)$ & $0 / 1(0 / 7.1)$ & 0/NA (0/NA) & $6(0.68)$ \\
\hline Numbness & $0 / 0(0 / 0)$ & $2 / 2(0.5 / 0.5)$ & $0 / 0(0 / 0)$ & 0/NA (0/NA) & $4(0.45)$ \\
\hline Panic attack & $0 / 0(0 / 0)$ & $1 / 3(0.3 / 0.8)$ & $0 / 0(0 / 0)$ & 0/NA (0/NA) & $4(0.45)$ \\
\hline ENT symptoms & $0 / 0(0 / 0)$ & $1 / 2(0.3 / 0.5)$ & $0 / 0(0 / 0)$ & 0/NA (0/NA) & $3(0.34)$ \\
\hline Arrhythmias/BP & $0 / 0(0 / 0)$ & $1 / 2(0.3 / 0.5)$ & $0 / 0(0 / 0)$ & 0/NA (0/NA) & $3(0.34)$ \\
\hline Local swelling & $0 / 0(0 / 0)$ & $0 / 2(0 / 0.5)$ & $1 / 0(7.1 / 0)$ & 0/NA (0/NA) & $3(0.34)$ \\
\hline Lymphadenitis & $0 / 0(0 / 0)$ & $0 / 2(0 / 0.5)$ & $0 / 0(0 / 0)$ & 0/NA (0/NA) & $2(0.23)$ \\
\hline Leukopaenia & $0 / 0(0 / 0)$ & $1 / 1(0.3 / 0.3)$ & $0 / 0(0 / 0)$ & 0/NA (0/NA) & $2(0.23)$ \\
\hline Cough & $0 / 0(0 / 0)$ & $1 / 0(0.3 / 0)$ & $0 / 0(0 / 0)$ & 0/NA (0/NA) & $1(0.11)$ \\
\hline Pericarditis & $0 / 0(0 / 0)$ & $0 / 1(0 / 0.3)$ & $0 / 0(0 / 0)$ & 0/NA (0/NA) & $1(0.11)$ \\
\hline Itching & $0 / 0(0 / 0)$ & $0 / 1(0 / 0.3)$ & $0 / 0(0 / 0)$ & 0/NA (0/NA) & $1(0.11)$ \\
\hline GI symptomatology & $0 / 0(0 / 0)$ & $0 / 1(0 / 0.3)$ & $0 / 0(0 / 0)$ & 0/NA (0/NA) & $1(0.11)$ \\
\hline
\end{tabular}

\section{Disease flare}

Disease flare was reported from $1 / 441(0.23 \%)$ and $8 / 441$ $(1.81 \%)$ of the patients after the first and the second dose, respectively. Disease flare was not associated with any of the parameters tested, but marginally with the discontinuation of treatment due to COVID-19 vaccination [(2/21), 9.5\%, compared to those who did not discontinue their treatment for any reason, $6 / 356(1.7 \%), p=0.065]$. No variables were found to predict disease flare in the multivariable model employed. One should note though that a low number of patients were positive for the dependent variable (disease flare).

\section{Vaccine hesitancy and associating factors}

For those who declined vaccination, the vaccine offered was: Pfizer in 61 (50.8\%), Moderna in $12(10.0 \%)$, AstraZeneca in $1(0.8 \%)$ and no specific vaccine in $56(46.7 \%)$ patients. The reasons for non-vaccination against COVID19 were as follows: the vaccine would not be helpful $(15 / 120,12.5 \%)$, the vaccine would be harmful $(64 / 120$, $53.3 \%)$, could cause disease flare $(29 / 120,24.2 \%)$ and $/$ or could cause thrombosis $(26 / 120,21.7 \%)$, vaccination against COVID-19 has not been suggested by their treating rheumatologist $(7 / 120,5.8 \%)$ and other reasons (e.g. allergies, treatment with rituximab; 15/120, 12.5\%).
Interestingly, almost half (40.7\%) of the patients reporting fear of thrombosis suffered from systemic lupus erythematosus (SLE) and/or primary or secondary antiphospholipid syndrome (APS). Importantly, 65/120 (54.2\%) answered that they would consider having the vaccine in the near future.

Non-vaccination uptake was associated with younger age (mean \pm SD $50.28 \pm 15.26$ years for unvaccinated and $55.52 \pm 14.48$ years for vaccinated, $p<0.0008)$ unemployment ( $p=0.023$ ) (Table 4) and co-existent diabetes mellitus ( $p=0.027$ ) or chronic obstructive pulmonary disease $(p=0.019)$ (Table 5). Furthermore, vaccination uptake for COVID-19 was significantly decreased in patients with negative vaccination and nocebo-prone behaviour (both for $p<0.0001$ ) (Table 5). It is also worth mentioning that previous COVID-19 infection was inversely associated with vaccination uptake $(p=0.015)$. Finally, no association was found between decreased COVID-19 vaccination uptake and co-medication (Table 4) or treatment non-adherence during the first wave of the COVID-19 pandemic (Table 5).

Multivariable analysis confirmed as independent variables: negative vaccination behaviour (Odds Ratio [OR]; 95\% confidence interval [CI], 6.56; 3.21-13.42), nocebo-prone behaviour (OR; 95\% CI, 3.88; 1.76-8.55), and previous COVID-19 infection (OR; 95\% CI, 2.83; 1.13-7.05), while university/college education was 
Table 4 Demographic and clinical characteristics of patients enrolled in our study
Table 5 Comorbidities and behavioural patterns of patients enrolled in our study

\begin{tabular}{llll}
\hline Characteristics & Vaccinated $n=441$ & Non-vaccinated $N=120$ & $p$ value \\
\hline Female gender, $n(\%)$ & $335(75.9)$ & $89(74.2)$ & 0.719 \\
Age, mean \pm SD & $55.52 \pm 14.48$ & $50.28 \pm 15.26$ & $\mathbf{0 . 0 0 0 8}$ \\
Smoking (current), $n(\%)$ & $122(27.7)$ & $29(24.2)$ & 0.487 \\
Disease & & & 0.668 \\
Inflammatory arthritis, $n(\%)$ & $258(58.51)$ & $68(56.7)$ & \\
Connective tissue diseases, $n(\%)$ & $118(26.8)$ & $36(30.0)$ & \\
Autoinflammatory, $n(\%)$ & $16(3.6)$ & $6(5.0)$ & 0.965 \\
Vasculitis, $n(\%)$ & $49(11.1)$ & $10(8.3)$ & 0.167 \\
Disease duration (years), mean \pm SD & $9.9 \pm 8.3$ & $10.3 \pm 9.1$ & $\mathbf{0 . 0 2 3}$ \\
College/university level of education, $n(\%)$ & $172(39.0)$ & $38(31.7)$ & \\
Unemployment, $n(\%)$ & $72(16.3)$ & $31(25.8)$ & 0.282 \\
Treatment & & & 0.915 \\
On steroids, $n(\%)$ & $151(34.2)$ & $48(40.0)$ & 0.917 \\
On cDMARDs, $n(\%)$ & $287(65.1)$ & $77(64.2)$ & $68(56.7)$ \\
On b/tsDMARDs, $n(\%)$ & $245(55.5)$ & & \\
\hline
\end{tabular}

Comparison between vaccinated and non-vaccinated patients

$S D$ standard deviation, $n$ number, $c D M A R D S$ conventional disease-modifying antirheumatic drugs, $b / t s D$ MARDs biologic or targeted synthetic DMARDs

\begin{tabular}{lllc}
\hline Characteristics & Vaccinated, $n=437$ & $\begin{array}{l}\text { Non-vaccinated, } \\
N=120\end{array}$ & $p$ value \\
\hline Arterial hypertension, $n(\%)$ & $139(31.8)$ & $29(24.2)$ & 0.144 \\
Coronary heart disease, $n(\%)$ & $27(6.2)$ & $5(4.2)$ & 0.510 \\
Congestive heart failure, $n(\%)$ & $5(1.1)$ & $2(1.7)$ & 0.645 \\
Diabetes mellitus, $n(\%)$ & $36(8.2)$ & $3(2.5)$ & $\mathbf{0 . 0 2 7}$ \\
Chronic kidney disease, $n(\%)$ & $31(7.1)$ & $8(6.7)$ & 1.000 \\
Chronic obstructive pulmonary disease, $n(\%)$ & $19(4.3)$ & $0(0.0)$ & $\mathbf{0 . 0 1 9}$ \\
Missed appointment (during last year), $n(\%)$ & $169(38.7)$ & $54(45.0)$ & 0.207 \\
Treatment discontinuation amid first COVID-19 & $6 / 250(2.4)$ & $2 / 73(2.7)$ & 1.000 \\
$\quad$ wave, $n(\%)$ & & & \\
Nocebo behaviour, $n(\%)^{\mathrm{a}}$ & $23 / 352(6.5)$ & $47 / 65(27.7)$ & $\mathbf{0 . 0 0 0 1}$ \\
Negative vaccination behaviour, $n(\%)^{\mathrm{a}}$ & $30(6.9)$ & & $\mathbf{0 . 0 0 0 1}$ \\
\hline
\end{tabular}

Comparison between vaccinated and unvaccinated patients

aFor definitions please see "Patients and methods" negatively associated with decreased uptake of COVID19 vaccine (OR; 95\% CI, 0.49; 0.26-0.92).

\section{Discussion}

Herein, we describe vaccination safety and reasons for vaccine hesitancy in a large cohort of patients with SRD. This is the largest study, conducted via interview with SRD patients. Of note, our study took place in a period in which vaccination against COVID-19 has been offered to all individuals with SRD. We found that $75 \%$ of patients with SRDs were vaccinated against COVID-19, with the majority receiving an mRNA-based vaccine. In accordance with a similar study using different methodology (online questionnaire sent to the patients) [14], about $35 \%$ of the patients in our cohort reported AEs to the vaccination.

AEs rates were comparable across different types of vaccines. For the first time, we report that the rate of AEs was similar between the first and second dose of vaccination for mRNA vaccines, but double for the first compared to the second dose for the Astra-Zeneca vaccine. To be mentioned, 
only two of our patients received the Johnson \& Johnson vaccine, therefore no definite conclusion can be drawn for this vaccine. In agreement with previously published studies focusing on mRNA vaccines, we found that fatigue and local pain at the site of injection were the most common symptoms for Pfizer vaccine $[13,19,20]$. In our cohort, fever was the cardinal AE for Astra-Zeneca vaccine, while myalgias and headache were not so commonly reported as in other studies [19]. Looking for interactions with clinical, demographic and other characteristics, we are in concert with another study finding that AEs are more common in females [14], reporting also for the first time that the occurrence of COPD is also a risk factor.

With this study, we also add to the accumulating evidence that vaccination against COVID-19 does not seem to be associated with exacerbation of SRDs, at least in the short term [3, 14, 15]. It should be mentioned though that disease flare is not formally assessed in most of the studies. Also, different time points of disease flare assessment have been used. Of note, most of disease flares seem to occur within the first 7 days after the vaccine [21]. These could explain some discrepancies observed in the percentages reported [21]. Importantly, we found that stopping treatment for COVID-19 vaccination was marginally associated with disease flare. This highlights the need for more studies that could support the recommendations issued about withholding some of the immunomodulatory drugs in the period around vaccination [22].

In accordance with previous studies which explored patients' concerns before COVID-19 vaccination programme expands $[10,12]$, and with more recent studies examining vaccine hesitancy in SRD patients [23], we found that fear of side effects as well as fear of disease flare is the major cause that leads to decreased vaccination uptake.

Analysing also clinical and demographic data, we showed that vaccination was less attractive for patients that did not have university/college education. This is in line with a recently published study with similar setting [23], as well as with the emerging concept of "syndemics" which highlights that all aspects (including social) should be taken into account in the treatment of SRD patients, especially in the COVID-19 era [24, 25]. Additionally, negative vaccination behaviour (defined here as not being vaccinated against influenza for the last 2 years), as well as nocebo-prone behaviour, has been strongly associated in our study with decreased rates of vaccination against COVID-19. Notably, another study from our department has shown that nocebo-prone behaviour assessed also by Q-No was associated with vaccine hesitancy in healthcare workers [26]. Nocebo effect is an increasingly recognized problem in patients with autoimmune diseases [27], which is more pronounced during COVID-19 era [17]. Noteworthy, earlier study from our department showed that despite influenza vaccination rates increased amid COVID-19 pandemic, there is still room for further improvement [28]. Besides, Felten et al. showed earlier this year that willingness for vaccination was higher in patients who received influenza or pneumococcal vaccine over the last years [10]. Similar findings were reported by Priori et al. in an online survey, which has shown that patients were willing to reconsider if medical education was provided [12]. In line with this finding, more than half of the unvaccinated patients in our study were willing to reconsider their decision. Another study has described in more detail the thoughts that might make patients hesitant/suspicious of the COVID-19 vaccine [29]. To this end, patients' and clinicians' education about benefits of vaccination is of paramount importance.

Interestingly, similar to other investigators [12, 29], we did not find any association between vaccination uptake and comorbidities and/or treatment in our cohort. On the other hand, in accordance with other investigators [23, 30], we found that increased age was associated with willingness to vaccinate against COVID-19. No association was found between decreased vaccination rate and missed appointments with the rheumatology team over the last year. This could imply that telemedicine worked well over the last 12 months, providing support to SRD patients. Indeed, the majority have been in telephone contact with the rheumatology team. Also, we found that patients who discontinued their treatment due to COVID-19 last year [18] were now vaccinated, showing the benefits from telephone communication. Finally, we should delineate that fear for thrombosis is still here, without being associated with a specific type of vaccine. Instead, we showed that the majority of patients expressing this concern were those with SLE and/or APS, as expected. This implies that we should probably focus more on this subgroup of patients, explaining that there is no additional risk for them [31].

Finally, although assessment of COVID-19 vaccination efficacy was beyond the scopes of this study, we observed (data not shown) that individuals vaccinated were less commonly infected (8.4\%), compared to those who were unvaccinated $(16.4 \%, p<0.0001)$. Notably, in studies exploring immunogenicity of vaccines in SRD patients, $5-10 \%$ did not produce adequate titers of antibodies, which is in line with the percentage of patients in our study who were infected without vaccination $[4,6,7]$.

We acknowledge that our study has some limitations. First, it is a retrospective study based on telephone interviews. However, all of them were conducted within 4 months after vaccination and by an experienced interviewer. We used this methodology, instead of online questionnaires to reduce the self-reporting bias. Also, we do not have an arm with people without SRD in our study; other studies have shown that AEs are largely similar 
between SRD and healthy individuals [3, 14, 15]. Notably, the percentage of vaccine hesitancy among SRD is similar to that reported for white British healthcare workers [32]. Additionally, nocebo effect was measured with the Q-No questionnaire. Despite not being validated extensively, it has been used in populations with SRD [17] and beyond [33]. Finally, the severity of reported AEs was not recorded, although no AE led to hospitalization.

On the other hand, the strengths of our study include that we enrolled patients with many different SRDs, taking also into account social and other clinical determinants like unemployment, educational status and comorbidities, respectively. Also, during the study period, vaccination against COVID-19 had been offered to all patients with SRD and three different vaccines have been employed. Finally, we examined the general behaviour against vaccines having as "pilot" the vaccination uptake against influenza for the last 2 years. In fact, our study suggests that vaccination campaign should focus on SRD patients with nocebo-prone and negative vaccination behaviour.

In conclusion, we showed that AEs are present in about one-third of the patients, more commonly observed in females and those with COPD. Minor differences exist in the type and rate of AEs across different vaccine types. The underlying disease activity remained unchanged and vaccination was highly protective against COVID-19. On the other hand, we showed that fear for vaccine against COVID-19 is still evident. This is more pronounced in patients with negative thoughts against vaccines, noceboprone behaviour, lower educational level, as well as in those previously infected with COVID-19. As more than half of the unvaccinated individuals in our study would reconsider being vaccinated, it seems that there is still room for improvement of vaccination rates in patients with SRD.) Thus, the vaccination campaign should go on and inform patients about the safety of vaccination against COVID-19.

Author contributions Data acquisition: GEF, VKB, EM, GE, KF, AK, EK, KL, SP, MP. Data analysis: GEF, VKB. Data interpretation: GEF, VKB, EM, GE, KF, AK, EK, KL, SP, MP, DDK, MGT, CV, PPS. Drafting manuscript: GEF, VKB, PPS. Revising manuscript: all authors.

Funding No specific funding has been received for this study.

Availability of data and material Raw data are available upon request.

Code availability Not applicable.

\section{Declarations}

Conflict of interest Nothing to declare.
Ethics approval This study was approved by the Scientific Council of our hospital (PN: 4/2020).

Consent to participate Informed consent (verbal due to nature of the study) has been obtained from all participants.

Consent for publication Not applicable.

\section{References}

1. Strangfeld A, Schafer M, Gianfrancesco MA, Lawson-Tovey S, Liew JW, Ljung L, Mateus EF, Richez C, Santos MJ, Schmajuk G, Scire CA, Sirotich E, Sparks JA, Sufka P, Thomas T, Trupin L, Wallace ZS, Al-Adely S, Bachiller-Corral J, Bhana S, Cacoub P, Carmona L, Costello R, Costello W, Gossec L, Grainger R, Hachulla E, Hasseli R, Hausmann JS, Hyrich KL, Izadi Z, Jacobsohn L, Katz P, Kearsley-Fleet L, Robinson PC, Yazdany J, Machado PM, Alliance C-GR (2021) Factors associated with COVID-19-related death in people with rheumatic diseases: results from the COVID-19 Global Rheumatology Alliance physician-reported registry. Ann Rheum Dis 80:930-942. https://doi. org/10.1136/annrheumdis-2020-219498

2. Benucci M, Infantino M, Marotto D, Ardizzone S, Manfredi M, Sarzi-Puttini P (2021) Vaccination against SARS-CoV-2 in patients with rheumatic diseases: doubts and perspectives. Clin Exp Rheumatol 39:196-202

3. Furer V, Eviatar T, Zisman D, Peleg H, Paran D, Levartovsky D, Zisapel M, Elalouf O, Kaufman I, Meidan R, Broyde A, Polachek A, Wollman J, Litinsky I, Meridor K, Nochomovitz H, Silberman A, Rosenberg D, Feld J, Haddad A, Gazzit T, Elias M, Higazi N, Kharouf F, Shefer G, Sharon O, Pel S, Nevo S, Elkayam O (2021) Immunogenicity and safety of the BNT162b2 mRNA COVID-19 vaccine in adult patients with autoimmune inflammatory rheumatic diseases and in the general population: a multicentre study. Ann Rheum Dis 80:1330-1338. https://doi.org/10.1136/annrh eumdis-2021-220647

4. Geisen UM, Berner DK, Tran F, Sumbul M, Vullriede L, Ciripoi M, Reid HM, Schaffarzyk A, Longardt AC, Franzenburg J, Hoff P, Schirmer JH, Zeuner R, Friedrichs A, Steinbach A, Knies C, Markewitz RD, Morrison PJ, Gerdes S, Schreiber S, Hoyer BF (2021) Immunogenicity and safety of anti-SARS-CoV-2 mRNA vaccines in patients with chronic inflammatory conditions and immunosuppressive therapy in a monocentric cohort. Ann Rheum Dis 80:1306-1311. https://doi.org/10.1136/annrh eumdis-2021-220272

5. Ramirez GA, Della-Torre E, Moroni L, Yacoub MR, Dagna L, Group O-Cs (2021) Correspondence on "Immunogenicity and safety of anti-SARS-CoV-2 mRNA vaccines in patients with chronic inflammatory conditions and immunosuppressive therapy in a monocentric cohort." Ann Rheum Dis 80:e159. https://doi. org/10.1136/annrheumdis-2021-220539

6. Ruddy JA, Connolly CM, Boyarsky BJ, Werbel WA, ChristopherStine L, Garonzik-Wang J, Segev DL, Paik JJ (2021) High antibody response to two-dose SARS-CoV-2 messenger RNA vaccination in patients with rheumatic and musculoskeletal diseases. Ann Rheum Dis 80:1351-1352. https://doi.org/10.1136/annrh eumdis-2021-220656

7. Veenstra J, Wang J, McKinnon-Maksimowicz K, Liu T, Zuniga B, Hamzavi I, Zhou L, Mi QS (2021) Correspondence on "Immunogenicity and safety of anti-SARS-CoV-2 mRNA vaccines in patients with chronic inflammatory conditions and immunosuppressive therapy in a monocentric cohort." Ann Rheum Dis 80:e160. https://doi.org/10.1136/annrheumdis-2021-220736 
8. Machingaidze S, Wiysonge CS (2021) Understanding COVID19 vaccine hesitancy. Nat Med 27:1338-1339. https://doi.org/10. 1038/s41591-021-01459-7

9. MacDonald NE, Hesitancy SWGoV (2015) Vaccine hesitancy: definition, scope and determinants. Vaccine 33:4161-4164. https://doi.org/10.1016/j.vaccine.2015.04.036

10. Felten R, Dubois M, Ugarte-Gil MF, Chaudier A, Kawka L, Bergier H, Costecalde C, Pijnenburg L, Fort J, Chatelus E, Sordet C, Javier RM, Gottenberg JE, Sibilia J, Fuentes-Silva Y, Arnaud L (2021) Vaccination against COVID-19: Expectations and concerns of patients with autoimmune and rheumatic diseases. Lancet Rheumatol 3:e243-e245. https://doi.org/10.1016/S2665-9913(21) 00039-4

11. Furer V, Rondaan C, Agmon-Levin N, van Assen S, Bijl M, Kapetanovic MC, de Thurah A, Mueller-Ladner U, Paran D, Schreiber K, Warnatz K, Wulffraat NM, Elkayam O (2021) Point of view on the vaccination against COVID-19 in patients with autoimmune inflammatory rheumatic diseases. RMD Open 7:e01594. https://doi.org/10.1136/rmdopen-2021-001594

12. Priori R, Pellegrino G, Colafrancesco S, Alessandri C, Ceccarelli F, Di Franco M, Riccieri V, Scrivo R, Sili Scavalli A, Spinelli FR, Conti F (2021) SARS-CoV-2 vaccine hesitancy among patients with rheumatic and musculoskeletal diseases: a message for rheumatologists. Ann Rheum Dis 80:953-954. https://doi.org/10.1136/ annrheumdis-2021-220059

13. Connolly CM, Ruddy JA, Boyarsky BJ, Avery RK, Werbel WA, Segev DL, Garonzik-Wang J, Paik JJ (2021) Safety of the first dose of mRNA SARS-CoV-2 vaccines in patients with rheumatic and musculoskeletal diseases. Ann Rheum Dis. https://doi.org/10. 1136/annrheumdis-2021-220231

14. Boekel L, Kummer LY, van Dam KPJ, Hooijberg F, van Kempen Z, Vogelzang EH, Wieske L, Eftimov F, van Vollenhoven R, Kuijpers TW, van Ham SM, Tas SW, Killestein J, Boers M, Nurmohamed MT, Rispens T, Wolbink G (2021) Adverse events after first COVID-19 vaccination in patients with autoimmune diseases. Lancet Rheumatol 3:e542-e545. https://doi.org/10.1016/ S2665-9913(21)00181-8

15. Cherian S, Paul A, Ahmed S, Alias B, Manoj M, Santhosh AK, Varghese DR, Krishnan N, Shenoy P (2021) Safety of the ChAdOx1 nCoV-19 and the BBV152 vaccines in 724 patients with rheumatic diseases: a post-vaccination cross-sectional survey. Rheumatol Int 41:1441-1445. https://doi.org/10.1007/ s00296-021-04917-0

16. Mitsikostas DD, Deligianni CI (2015) Q-No: a questionnaire to predict nocebo in outpatients seeking neurological consultation. Neurol Sci 36:379-381. https://doi.org/10.1007/ s10072-014-1959-2

17. Fragoulis GE, Evangelatos G, Arida A, Bournia VK, Fragiadaki K, Karamanakos A, Kravvariti E, Laskari K, Panopoulos S, Pappa M, Mitsikostas DD, Tektonidou MG, Sfikakis PP (2020) Noceboprone behaviour in patients with autoimmune rheumatic diseases during the COVID-19 pandemic. Mediterr J Rheumatol 31:288294. https://doi.org/10.31138/mjr.31.3.288

18. Fragoulis GE, Evangelatos G, Arida A, Bournia VK, Fragiadaki K, Karamanakos A, Kravvariti E, Laskari K, Panopoulos S, Pappa M, Tektonidou MG, Sfikakis PP (2020) Treatment adherence of patients with systemic rheumatic diseases in COVID19 pandemic. Ann Rheum Dis. https://doi.org/10.1136/annrh eumdis-2020-217935

19. Esquivel-Valerio JA, Skinner-Taylor CM, Moreno-Arquieta IA, Cardenas-de la Garza JA, Garcia-Arellano G, Gonzalez-Garcia PL, Almaraz-Juarez FDR, Galarza-Delgado DA (2021) Adverse events of six COVID-19 vaccines in patients with autoimmune rheumatic diseases: a cross-sectional study. Rheumatol Int. https:// doi.org/10.1007/s00296-021-05017-9
20. Bartels LE, Ammitzboll C, Andersen JB, Vils SR, Mistegaard CE, Johannsen AD, Hermansen MF, Thomsen MK, Erikstrup C, Hauge EM, Troldborg A (2021) Local and systemic reactogenicity of COVID-19 vaccine BNT162b2 in patients with systemic lupus erythematosus and rheumatoid arthritis. Rheumatol Int 41:1925-1931. https://doi.org/10.1007/s00296-021-04972-7

21. Barbhaiya M, Levine JM, Bykerk VP, Jannat-Khah D, Mandl LA (2021) Systemic rheumatic disease flares after SARS-CoV-2 vaccination among rheumatology outpatients in New York City. Ann Rheum Dis 80:1352-1354. https://doi.org/10.1136/annrh eumdis-2021-220732

22. Curtis JR, Johnson SR, Anthony DD, Arasaratnam RJ, Baden LR, Bass AR, Calabrese C, Gravallese EM, Harpaz R, Kroger A, Sadun RE, Turner AS, Williams EA, Mikuls TR (2021) American College of Rheumatology Guidance for COVID-19 vaccination in patients with rheumatic and musculoskeletal diseases: version 2. Arthritis Rheumatol 73:e30-e45. https://doi.org/10.1002/art. 41877

23. Gaur P, Agrawat H, Shukla A (2021) COVID-19 vaccine hesitancy in patients with systemic autoimmune rheumatic disease: an interview-based survey. Rheumatol Int 41:1601-1605. https:// doi.org/10.1007/s00296-021-04938-9

24. Nikiphorou E, Alpizar-Rodriguez D, Gastelum-Strozzi A, Buch M, Pelaez-Ballestas I (2021) Syndemics \& syndemogenesis in COVID-19 and rheumatic and musculoskeletal diseases: old challenges, new era. Rheumatology (Oxford) 60:2040-2045. https:// doi.org/10.1093/rheumatology/keaa840

25. Nikiphorou E, Lempp H, Kohrt BA (2019) Treatment failure in inflammatory arthritis: time to think about syndemics? Rheumatology (Oxford) 58:1526-1533. https://doi.org/10.1093/rheum atology/kez222

26. Mitsikostas DD, Aravantinou-Fatorou KDC, Kravvariti EKE, Mylona MVP, Papagiannopoulou GDE-M, Dellis ATG, Dimopoulos MA, Amanzio M, Sfikakis PP (2021) Nocebo-prone behavior associated with SARS-CoV-2 vaccine hesitancy in healthcare workers. Vaccines. https://doi.org/10.3390/vaccines9101179

27. Kravvariti E, Kitas GD, Mitsikostas DD, Sfikakis PP (2018) Nocebos in rheumatology: emerging concepts and their implications for clinical practice. Nat Rev Rheumatol 14:727-740. https://doi. org/10.1038/s41584-018-0110-9

28. Fragoulis GE, Grigoropoulos I, Mavrea E, Arida A, Bournia VK, Evangelatos G, Fragiadaki K, Karamanakos A, Kravvariti E, Panopoulos S, Pappa M, Thomas K, Tektonidou MG, Paraskevis D, Vassilopoulos D, Sfikakis PP (2021) Increased influenza vaccination rates in patients with autoimmune rheumatic diseases during the COVID-19 pandemic: a cross-sectional study. Rheumatol Int 41:895-902. https://doi.org/10.1007/s00296-021-04817-3

29. Felten R, Dubois M, Ugarte-Gil MF, Chaudier A, Kawka L, Bergier H, Costecalde C, Pijnenburg L, Fort J, Chatelus E, Sordet C, Javier RM, Gottenberg JE, Sibilia J, Fuentes-Silva YJ, Arnaud L (2021) Cluster analysis reveals 3 main patterns of behavior towards SARS-CoV-2 vaccination in patients with autoimmune and inflammatory diseases. Rheumatology (Oxford) 60:S168 S176. https://doi.org/10.1093/rheumatology/keab432

30. Yurttas B, Poyraz BC, Sut N, Ozdede A, Oztas M, Ugurlu S, Tabak F, Hamuryudan V, Seyahi E (2021) Willingness to get the COVID-19 vaccine among patients with rheumatic diseases, healthcare workers and general population in Turkey: a web-based survey. Rheumatol Int 41:1105-1114. https://doi.org/10.1007/ s00296-021-04841-3

31. Felten R, Kawka L, Dubois M, Ugarte-Gil MF, Fuentes-Silva Y, Piga M, Arnaud L (2021) Tolerance of COVID-19 vaccination in patients with systemic lupus erythematosus: the international VACOLUP study. Lancet Rheumatol 3:e613-e615. https://doi.org/ 10.1016/S2665-9913(21)00221-6 
32. Woolf K, McManus IC, Martin CA, Nellums LB, Guyatt AL, Melbourne C, Bryant L, Gogoi M, Wobi F, Al-Oraibi A, Hassan O, Gupta A, John C, Tobin MD, Carr S, Simpson S, Gregary B, Aujayeb A, Zingwe S, Reza R, Gray LJ, Khunti K, Pareek M, Group U-RSC (2021) Ethnic differences in SARS-CoV-2 vaccine hesitancy in United Kingdom healthcare workers: Results from the UK-REACH prospective nationwide cohort study. Lancet Reg Health Eur. https://doi.org/10.1016/j.lanepe.2021.100180

33. Mitsikostas DD, Belesioti I, Arvaniti C, Mitropoulou E, Deligianni C, Kasioti E, Constantinidis T, Dermitzakis M, Vikelis M,
Hellenic Headache S (2017) Patients' preferences for headache acute and preventive treatment. J Headache Pain 18:102. https:// doi.org/10.1186/s10194-017-0813-3

Publisher's Note Springer Nature remains neutral with regard to jurisdictional claims in published maps and institutional affiliations.

\section{Authors and Affiliations}

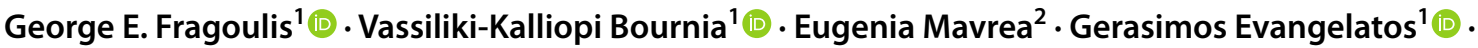 Kalliopi Fragiadaki ${ }^{1}$ - Anastasios Karamanakos ${ }^{1}$ - Eyrydiki Kravariti ${ }^{1} \cdot$ Katerina Laskari $^{1}$ (D) Stylianos Panopoulos ${ }^{1}$ (i) . Maria Pappa ${ }^{1}$ - Dimos D. Mitsikostas ${ }^{3}$ (D) - Maria G. Tektonidou ${ }^{1}$ (I) - Dimitrios Vassilopoulos ${ }^{2}$ (D) Petros P. Sfikakis ${ }^{1}$ (I)}

\author{
Vassiliki-Kalliopi Bournia \\ lily_bournia@hotmail.com \\ Eugenia Mavrea \\ jenny.mavrea96@gmail.com \\ Gerasimos Evangelatos \\ gerevag@gmail.com \\ Kalliopi Fragiadaki \\ po_fragiadaki@yahoo.gr \\ Anastasios Karamanakos \\ t_kar84@gmail.com \\ Eyrydiki Kravariti \\ euridicek@gmail.com \\ Katerina Laskari \\ katerina_laskari@yahoo.gr \\ Stylianos Panopoulos \\ sty.panopoulos@gmail.com \\ Maria Pappa \\ mariak.pappa@yahoo.com
}

Dimos D. Mitsikostas

dimosmitsikostas@me.com

Maria G. Tektonidou

mtektonidou@gmail.com

Dimitrios Vassilopoulos

dvassilop@med.uoa.gr

Petros P. Sfikakis

psfikakis@med.uoa.gr

1 Joint Rheumatology Program, Rheumatology Unit, First Department of Propaedeutic Internal Medicine, Athens School of Medicine, National and Kapodistrian University, Mikras Asias 75 str, 11527 Athens, Greece

2 2nd Department of Medicine and Laboratory, Clinical Immunology-Rheumatology Unit, Hippokration General Hospital, Athens, Greece

3 1st Neurology Department, Medical School, Aeginition Hospital, National and Kapodistrian University of Athens, Athens, Greece 